physicians' outcomes and events, patients are empowered to make more informed decisions when choosing their doctor. Such a registry helps to redress the information asymmetry found at the core of the decision-making problem for patients when attempting to choose their doctor. Examples of PROPER registries include the NICOR Adult PCI Registry in the UK which reports Percutaneous Coronary Intervention (PCI) outcomes for all operators in the UK. Another example (since 1991) is the New York State Cardiac Registry which publishes risk adjusted outcome statistics for all cardiothoracic surgeons operating in the State of New York. Currently, there are no PROPER registries in Ireland. There has only been publication of institution level outcomes through the National Healthcare Quality Reporting System (NHQRS). We surveyed practicing cardiologists and trainees in Ireland, the aim was to examine their perception of the benefits and potential harms associated with PROPER registries. The potential impact such registries might have on referral patterns, market share, how patients choose their cardiologist and whether risk adjustment algorithms are sufficient to protect against adverse behaviours.

Methods Data was collected by means of a 20-question survey circulated amongst attendees at the Irish Cardiac Society (ICS) Meeting 2019. An electronic copy of the survey was also distributed to cardiology practitioners and trainees known to the author but not encountered at the ICS conference. The questions (see table 1) were framed as statements, respondents were then asked to quantify their agreement or disagreement with the statement based on a 5 -point Likert scale.

Results A total of 39 responses to the survey were received (estimate $26 \%$ of all possible responses). Irish cardiologists are in general optimistic regarding the potential benefits of PROPER. Most markedly at an institution level, 81\% agreeing that it would improve patient care. $87 \%$ of respondents felt that referrals would be significantly affected by public reporting. $77 \%$ felt PROPER would impact market share of public versus private practice. $60 \%$ of responders considered mortality to be an appropriate quality assurance metric for cardiology (in contrast to only $15 \%$ found in similar studies). An overwhelming 97\% of respondents felt that public reporting would affect doctors' willingness to intervene on high risk patients. $87 \%$ of respondents felt that PROPER would lead to other adverse behaviours such as 'upcoding' or 'gaming.'

Conclusion In conclusion we see that Irish cardiologists in general hold an optimistic view regarding the potential benefits of PROPER. However, they are also aware of its potential for adverse unintended consequences. Responses to this survey were broadly in keeping with those founds in other studies performed in jurisdictions with active PROPER registries, though there were some notable differences.

\section{USE OF ULTRASOUND DERIVED CAROTID INTIMA- MEDIA THICKNESS AND PLAQUE VOLUME TO PREDICT SINGLE OR MULTIVESSEL CORONARY ARTERY DISEASE}

${ }^{1} \mathrm{~K}$ Owen, ${ }^{1}$ I Menown, ${ }^{2} \mathrm{~J}$ McLaughlin. ${ }^{1}$ Craigavon Area Hospital, Craigavaon, UK; ${ }^{2}$ Ulster University, UK

10.1136/heartjnl-2020-ICS.34
Background Increased carotid intima-media thickness (cIMT) has been extensively evaluated as a marker of cardiovascular (CV) risk. However, there is only limited evidence correlating cIMT measurements with anatomical severity of vascular disease. This study compared the value cIMT \pm other carotid measurements to predict the presence and severity of significant coronary artery disease (CAD).

Methods Patients were included with a history of ischaemictype chest pain or angina equivalent and undergoing either Invasive Coronary Angiogram (ICA) or Computed Tomography Coronary Angiography (CTCA). All patients underwent high-resolution B-mode ultrasound imaging to measure cIMT and B-Mode 3D-imaging to measure Total Plaque Volume (TPV) and Maximum Area Reduction (MAR) using latest generation dedicated hardware and software. cIMT normal ranges were defined by previous independent population studies. An operator, blinded to carotid measurements, defined the presence and severity CAD on ICA or CTCA. The study was supported by a European Union INTERREG VA Programme grant.

Results The study population comprised of 90 subjects $(73 \%$ male). Mean age was $66.2 \pm 11.63 \mathrm{SD}$ years (range, 42-88 years). CV risks factors included family history of CAD (74\%), current or ex-smoker (69\%), hypertension (66\%), a history of hyperlipidaemia (61\%) and diabetes (21\%). On ICA or CTCA, $62 \%$ of patients had severe disease (defined as at least $70 \%$ area stenosis or pressure wire positive) in at least one coronary artery, and 39\% had severe multivessel disease (in 2 or more vessels). The presence of elevated cIMT $\geq 50$ th percentile predicted the likelihood of severe multivessel CAD (relative risk [RR] $1.49 ; 47 \%$ vs $21 \%$; $\mathrm{p}=0.022)$ and the likelihood of severe CAD in at least one vessel (RR $1.65 ; 71 \%$ vs $43 \% ; \mathrm{p}=0.011$ ). Use of cIMT $\geq 75$ th percentile cut off did not increase predictive value. On 3D analysis, TPV in the top tertile predicted likelihood of severe CAD in at least one vessel (RR 1.4; 77\% vs 55\%; $\mathrm{p}=0.046)$. MAR did not add further to the predictive value. (Table 1).

\begin{tabular}{|c|c|c|c|c|c|c|}
\hline & $\begin{array}{l}\text { CIMT } \\
\geq 50 \text { th } \\
\text { percentile } \\
(n=62)\end{array}$ & $\begin{array}{l}\text { CIMT } \\
<50 \text { th } \\
\text { percentile } \\
(n=28)\end{array}$ & $\begin{array}{l}\mathrm{p} \\
\text { value }\end{array}$ & $\begin{array}{l}\text { cIMT } \\
\geq 75 \text { th } \\
\text { percentile } \\
(\mathrm{n}=41)\end{array}$ & $\begin{array}{l}\text { clMT } \\
<75 \text { th } \\
\text { percentile } \\
(n=49)\end{array}$ & $\begin{array}{l}p \\
\text { value }\end{array}$ \\
\hline $\begin{array}{l}\text { Severe CAD } \\
\text { in at least } \\
\text { one vessel }\end{array}$ & 44 & 12 & 0.011 & 31 & 25 & 0.016 \\
\hline $\begin{array}{l}\text { Severe } \\
\text { multivessel } \\
\text { CAD }\end{array}$ & 29 & 6 & 0.022 & 19 & 16 & 0.18 \\
\hline
\end{tabular}

Conclusions In a population of patients with ischaemic type chest pain or angina equivalent symptoms, elevated cIMT $(\geq 50$ th percentile) predicted an increased risk of severe CAD in at least one vessel and over double the risk of severe multivessel CAD. CIMT may thus be a useful tool to help triage patients most likely to benefit from invasive coronary investigation. 